\title{
Analysis of Microstructure and Low Permeability with 3D Digital Rock Modeling
}

\author{
Jinsui Wu $\mathbb{D}^{1,2}$ Min Xing ${ }^{10},{ }^{2}$ Shangxian Yin, ${ }^{2}$ Ciyuan Zhang, ${ }^{2}$ Xuexi Chen, ${ }^{2}$ Sihai Yi, \\ Huiqing Lian, ${ }^{2}$ and Dongyu Xie ${ }^{2}$ \\ ${ }^{1}$ State Key Laboratory of Oil and Gas Reservoir Geology and Exploitation, Southwest Petroleum University, \\ Chengdu 610500, China \\ ${ }^{2}$ Department of Safety Engineering, North China Institute of Science and Technology, Beijing 101601, China
}

Correspondence should be addressed to Jinsui Wu; 200700797wjs@ncist.edu.cn and Min Xing; 1765898497@qq.com

Received 8 October 2021; Revised 21 December 2021; Accepted 1 February 2022; Published 3 March 2022

Academic Editor: Steffen Berg

Copyright (c) 2022 Jinsui Wu et al. This is an open access article distributed under the Creative Commons Attribution License, which permits unrestricted use, distribution, and reproduction in any medium, provided the original work is properly cited.

The sandstone microstructure and permeability are important parameters for quantitative evaluation of groundwater/oil/gas resources and prediction of flow rates of water/oil/gas. In this study, we applied seven low-permeability sandstone samples obtained from North China to research the microstructure and permeability based on digital core technology. Rock images were collected by X-ray microcomputed tomography $(\mu \mathrm{CT})$, and then software (Avizo) was applied to analyze the microstructure and calculate the parameters such as porosity, connected porosity, average equivalent diameter, tortuosity, and shape factor. By introducing the shape factor into the Kozeny-Carman equation, we modified the Kozeny-Carman equation and found that the modified equation is a function of porosity, diameter of particles, tortuosity, and particle shape factor.

\section{Introduction}

The characteristics of low-permeability reservoirs are widely used to improve the water injection effect of lowpermeability oilfields or exploit oil and gas resources. The research of low permeability is essential to studying the reservoir characteristics. Reasonable characterization and quantitative analysis of the complex pore structures inside rock were the basis for solving many underground reservoir characteristics [1]. The research on the microstructure of the digital core was mainly based on the study of the 3D digital core. Knackstedt et al. [2] controlled the finite size effect, discretion error, and statistical fluctuations to obtain high-precision results by using X-micron CT scanners; Tian and Han [3] used X-ray CT to examine the evolution of concrete internal damage. Wang et al. [4] combined CT and the pore network model to analyze the influence of particle size on permeability in hydrate-containing porous media. Wang et al. [5] used fractal theory and X-ray CT imaging to carry out three-dimensional modeling and analysis of the coal pore structure. Cao et al. [6] used computed tomography data to analyze the capillary pressure of the dense sandstone based on a digital rock model. Qin et al. [7] used the fractal and multifractal methods such as box dimension and moment method combined with X-CT imaging techniques to analyze the structure of the volcanic vesicle. Yang et al. [8] used X-ray tomography to determine the distribution of residual oil in sandstone with different permeability.

Permeability was the key relationship between microstructure and macroscopic physical properties of porous media [9], and it was not only affected by the geometry or shape of the pore space but also by the topological structure [10]. The pore space connectivity of porous media was an important factor affecting the flow characteristics of tight sandstone [11]. The tortuosity of the pore structure has an important effect on macroscopic transport properties. Nakashima found that the diffusion rate and permeability decreased with the increase of tortuosity [12]. The pore size and mean coordination number also affected the overall permeability, and the permeability increased with the increase of the pore size distribution width $[13,14]$. Sueyoshi 
et al. also found that permeability mainly depends on the porosity and pore size of the flow path [15].

Various methods have been attempted to calculate permeability; $\mathrm{Hu}$ and Blunt used an improved maximum ball algorithm to study the overall network pore structure and calculate permeability [16]. A support vector regression (SVR) model was developed to evaluate the low porosity and permeability of sandstone reservoirs by Feng et al. [17]. Wei et al. [18] used the relevant parameters of the porous media to figure out the $\mathrm{KC}$ constant of the Kozeny-Carman equation, which thus contributes to the further study and analysis of the permeability. Kozeny-Carman (KC) equation expressed the relationship of permeability and porosity of porous media micropore structure [19-21]:

$$
k=\frac{\varphi^{3}}{c(1-\varphi)^{2} S^{2}}=\frac{\varphi^{3}}{36 c \tau^{2}(1-\varphi)^{2}} d^{2},
$$

where $k$ is the permeability; $\varphi$ is the porosity; $d$ is the average particle radius; $\tau$ is the tortuosity; and $c$ is the empirical constant.

However, the traditional Kozeny-Carman equation was an empirical formula. For a heterogeneous system, the calculated permeability was large, 10 or more times than the actual [22]. Many modified equations were presented. Pape et al. [23] proposed an equation for sand and gravel:

$$
k=\frac{\varphi r^{2}}{8 \tau^{2}}\left(\frac{2 \varphi}{3 \tau^{2}(1-\varphi)}\right)^{2 /\left(D_{f}-1\right)}
$$

where $r$ is the particle radius and $D_{f}$ is the fractal dimension of the medium.

Civan [24] proposed an equation for general porous media:

$$
\sqrt{\frac{k}{\varphi}}=\gamma\left(\frac{\varphi}{1-\varphi}\right)^{\beta}
$$
rocks:

Costa [25] proposed an expression suitable for porous

$$
k=C \frac{\varphi^{n}}{1-\varphi} .
$$

Based on the fractal theory, Yu [26] derived the permeability expression through a curved capillary model.

$$
k=\frac{\pi}{128} \frac{L_{0}^{1-D_{T}}}{A} \frac{P_{m}}{\Delta P} \frac{D_{f} \lambda_{\max }^{3+D_{T}}}{3+D_{T}-D_{f}},
$$

where $D_{T}$ is the tortuosity fractal dimension, $L_{0}$ is the characteristic length, and $\lambda_{\max }^{3+D_{T}}$ is the maximum pore diameter.

$\mathrm{Xu}$ and $\mathrm{Yu}$ [27] also established a modified K-C equation based on the fractal theory:

$$
k=C_{f}\left(\frac{\varphi}{1-\varphi}\right)^{\left(1+D_{T}\right) / 2} \lambda_{\max }^{2}
$$

Among them, $C_{f}$ is as follows:

$$
C_{f}=\frac{\left(\pi D_{f}\right)^{\left(1-D_{T}\right) / 2}\left[4\left(2-D_{f}\right)\right]^{\left(1-D_{T}\right) / 2}}{128\left(3+D_{T}-D_{f}\right)} .
$$

Karacan [28] used the fractal method to calculate the porosity and permeability of mining, and the permeability expression was as follows:

$$
\begin{aligned}
& k= \frac{\pi}{16 A_{T}} \frac{L_{0}^{1-D_{T}}}{\alpha} \frac{D_{p}}{2.531+D_{T}-D_{p}} \\
& \cdot \eta_{\max }^{2.531+D_{T}}\left[1-\left(\frac{\eta_{\min }}{\eta_{\max }}\right)^{2.531+D_{T}-D_{p}}\right],
\end{aligned}
$$

where $A_{T}$ is the total area; $\eta_{\max }$ is the maximum diameter of the pore; $\eta_{\min }$ is the minimum diameter of the pore; and $D_{p}$ is the fractal dimension of the pore.

Xia et al. [29] used twelve three-dimensional digital cores of sandstone reservoir rocks to evaluate permeability in terms of fractal dimension, lacunarity, and succolarity. The permeability was rewritten as follows:

$$
k=0.0239 e^{23.98 S U}
$$

where $S U$ is the succolarity, indicating the ability of the fluid to move in the porous medium.

Shen et al. [30] proposed the equivalent Kozeny-Carman equation to establish a permeability prediction model:

$$
k=\frac{\varphi^{3}}{180(1-\varphi)^{2}} d^{N} .
$$

In the formula, $N=2.44$, and the unit of $k$ is the same with the unit of $d^{2.44}$, i.e., $m^{2.44}$. This is different with the unit in the $\mathrm{KC}$ equation.

To sum up, digital rock cores (X-ray (CT) scanning) were used to obtain the structure of the rock cores, and some permeability prediction models have been proposed. The research was mainly on higher permeability, and few lowpermeability prediction models had been established. The low-permeability model generally contained empirical constants or modified permeability units.

This paper used X-ray CT scanning technology and Avizo software to study and calculate pore and particle structure parameters of low-permeability sandstones. Finally, the pore and particle structure parameters were used to predict permeability. In addition, the shape factor was introduced to modify the $\mathrm{KC}$ equation.

\section{The Porosity and Permeability Obtained by Experiments}

In this work, 7 natural sandstone samples were collected at depths of $430 \mathrm{~m}-735 \mathrm{~m}$ below the sea level of North China. These 7 natural sandstone samples were abbreviated as GQ1, GQ2, GQ3, LA1, LA2, TL1, and TL2. Samples GQ1, GQ2, and GQ3 were from Gequan Mine, samples LA1 and LA2 were from Lu'an Mine, and TL1 and TL2 were from Tunlan Mine. The permeability and porosity were tested using the core company's high- and low-permeability meter CAT112 
and a helium porosity meter from American Coretest company Phi220; the experiment results are shown in Table 1.

Based on the measured data, the map of sandstone porosity and permeability is shown in Figures 1 and 2 .

As can be seen from Figure 1, the porosity of sandstone in Gequan Mine is high, while the porosity of the Lu'an samples is relatively low. The highest porosity of Tunlan Mine (TL1) is $5.66 \%$, and the lowest porosity of Lu'an Mine (LA1) is $0.38 \%$.

Figure 2 shows the experiment permeability, and the permeability of Tunlan Mine (TL2) is the highest. Comparing Figures 1 and 2, it can be found that high porosity does not lead to high permeability; for example, the porosity of the samples GQ1, GQ2, GQ3, and TL1 is higher than that of other three samples (LA1, LA2, and TL2); however, the permeability values of the samples GQ1, GQ2, GQ3, TL1, LA1, and LA2 are relatively close, the porosity of Tunlan Mine (TL2) is $1.14 \%$, but the permeability is $0.0870 \mathrm{mD}$; this is higher than other samples.

\section{X-Ray Micro-CT Experiments and Calculations}

3.1. The Pore and Particle Diameters. This paper used a desktop micrometer CT scanner (NanoVoxel-3000) to measure samples of approximately $1 \mathrm{~cm}$. As an example, Figure 3 shows the sample GQ1 reconstructed images of three directions.

Avizo software was used to analyze the reconstructed 3D digital core. In order to better adjust the threshold so that the connected porosity is close to the measured porosity, a $3 \mathrm{D}$ digital core of $200 * 200 * 200$ was selected as the target study area which was larger than REV, and the voxel edge length was $18.05 \mu \mathrm{m}$. In addition, $200 * 200 * 200$ is also the study region in Figures 4-6.

Median filtering and interactive threshold segmentation were performed to obtain the three-dimensional pore structure of the core. Figure 4 shows the three-dimensional pore extraction process.

The pores of rock samples were divided into connected pores and isolated pores, i.e., total pores $=$ connected pores + isolated pores, as shown in Figure 5, the green parts are isolated pores, and the purple parts are connected pores. The GQ1 total porosity is $7.85 \%$, where the isolated porosity is $3.34 \%$ and the connected porosity is $4.51 \%$. It can be seen that the connected pore is about the experimentally measured data size. The porosity distribution of all rock samples is as follows (see Table 2).

To understand the distribution of pores with different pore sizes, the total pores were screened according to the pore size through the pore segmentation module of Avizo, and the pore size of the sample was obtained (as shown in Figure 6). The overall pore size distribution is shown in Figure 7.

Figure 7 shows that the $40-80 \mu \mathrm{m}$ diameters of the pore's aperture are the most widely distributed about $40 \%$. The proportion of large pores is relatively small, and the pores larger than $400 \mu \mathrm{m}$ are mainly connected pores. This was mainly due to the close connection of multiple pores, which resulted in the threshold segmentation as a whole. Finally, we obtained the sample GQ1's average pore diameter as $68 \mu \mathrm{m}$.

The particle part was obtained after removing the pores in the sample (see Figure 8). Through the analysis of diameters, we can see that the particles with a diameter of $0-25 \mu \mathrm{m}$ account for the largest proportion. The final analysis shows that the average particle diameter of the sample GQ1 is $35 \mu \mathrm{m}$. The diameter distributions of pores and particles for all rock samples are as follows (see Table 3).

3.2. Shape Factor Analysis of Rock Samples. In reality, not all the pores/capillaries are spherical, and the shape of the particles has an effect on the permeability [31, 32]. Nemec and Levec researched shape factor of trilobe and quadralobe particles and the effect on the permeability [33]. Safari et al. developed a porosity-permeability relationship for ellipsoidal grains [34].

We used the Label Analysis module of Avizo software (see Figure 9) to analyze the pores' shape factor. The calculation of the shape factor was based on the ideal sphere model. The calculation equation is

$$
\sigma=\frac{A^{3}}{36 \times \pi \times V^{2}},
$$

where $s$ is the shape factor, $A$ is the area, and $V$ is the volume of the pore.

Because of the presence of partially elongated pores, the surface area of the pore becomes larger, resulting in a shape factor greater than 1. According to the demarcation point of the shape factor, the pores' shape factor was divided; GQ1's image as a sample is shown in Figure 10. The shape factors of the pores and particles of the average sample GQ1 are 2.03 and 1.24 , respectively.

Figure 11 shows the statistic of the GQ1's pores and particles' shape factor distribution in each section.

Table 4 shows the average shape factor distribution of pores and particles for all rock samples.

3.3. Tortuosity Analysis of Rock Samples. The ball-and-stick model can intuitively show the connection among the pores. The GQ1's ball-and-stick model was established as shown in Figure 12.

The ball-and-stick model showed the connection between the internally connected pores in the rock sample. For this reason, the tortuosity can be analyzed by the ball-andstick model. Tortuosity $=$ path length/straight-line distance between two points.

$$
\tau=\frac{L_{t}}{L_{0}} .
$$

The tortuosity of the pore for all samples can be shown as follows (see Table 5). 
TABLE 1: Experiment results of porosity and permeability.

\begin{tabular}{lcccccccc}
\hline $\begin{array}{l}\text { Serial } \\
\text { number }\end{array}$ & $\begin{array}{c}\text { Sample } \\
\text { number }\end{array}$ & $\begin{array}{c}\text { Length } \\
(\mathrm{mm})\end{array}$ & $\begin{array}{c}\text { Diameter } \\
(\mathrm{mm})\end{array}$ & $\begin{array}{c}\text { Quality } \\
(\mathrm{g})\end{array}$ & $\begin{array}{c}\text { Confining pressure } \\
(\mathrm{psi})\end{array}$ & $\begin{array}{c}\text { Pore pressure } \\
(\mathrm{psi})\end{array}$ & $\begin{array}{c}\text { Porosity } \\
(\%)\end{array}$ & $\begin{array}{c}\text { Permeability } \\
(\mathrm{mD})\end{array}$ \\
\hline 1 & GQ1 & 35.70 & 25.73 & 45.44 & 560 & 200 & 4.42 & 0.0144 \\
2 & GQ2 & 28.63 & 25.45 & 36.19 & 560 & 200 & 3.88 \\
3 & GQ3 & 25.47 & 25.62 & 32.46 & 560 & 200 & 0.0102 \\
4 & TL1 & 37.94 & 25.67 & 48.18 & 560 & 200 & 5.58 & 0.0084 \\
5 & TL2 & 38.22 & 25.50 & 52.48 & 560 & 200 & 0.0073 \\
6 & LA1 & 37.88 & 25.69 & 53.39 & 560 & 200 & 0.14 \\
7 & LA2 & 36.65 & 25.53 & 49.44 & 560 & 200 & 0.0870 \\
\hline
\end{tabular}



FIGURE 1: Sandstone sample porosity distribution.

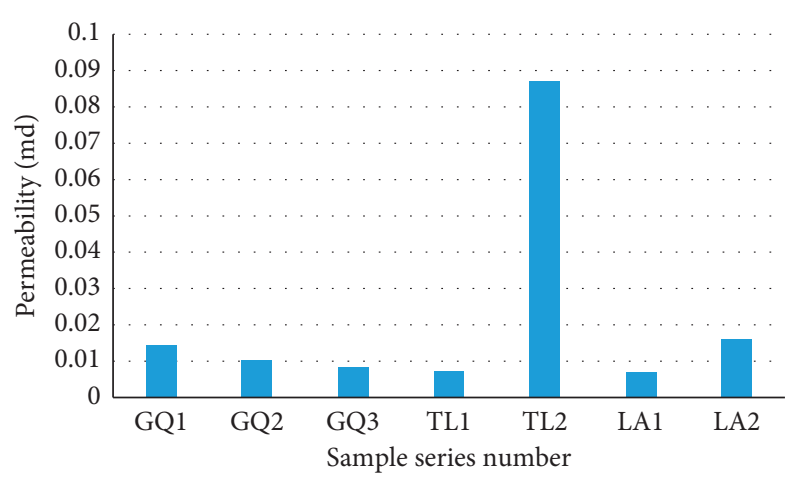

FIGURE 2: Sandstone sample permeability distribution.

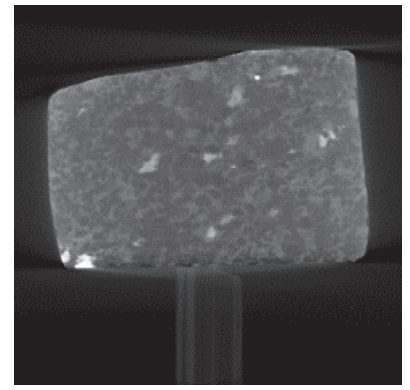

(a)

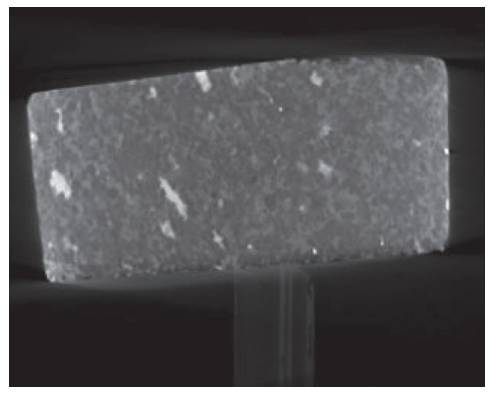

(b)

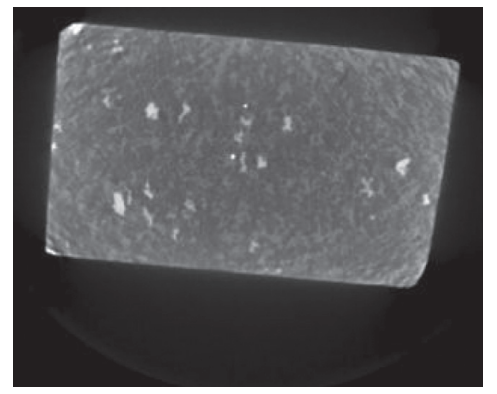

(c)

FIGURE 3: GQ1 reconstructed images of three directions. (a) $X$-axis direction. (b) $Y$-axis direction. (c) $Z$-axis direction.

\section{Permeability Prediction Model}

4.1. Kozeny-Carman Equation. The Kozeny-Carman equation, which was first proposed by Kozeny [19] in 1927 and revised by Carman [20, 21], was abbreviated as the $\mathrm{KC}$ equation. It was widely used in many fields to estimate and predict hydraulic conductivity, such as underground seepage, oil and gas field exploitation, chemical engineering, 



Target study area
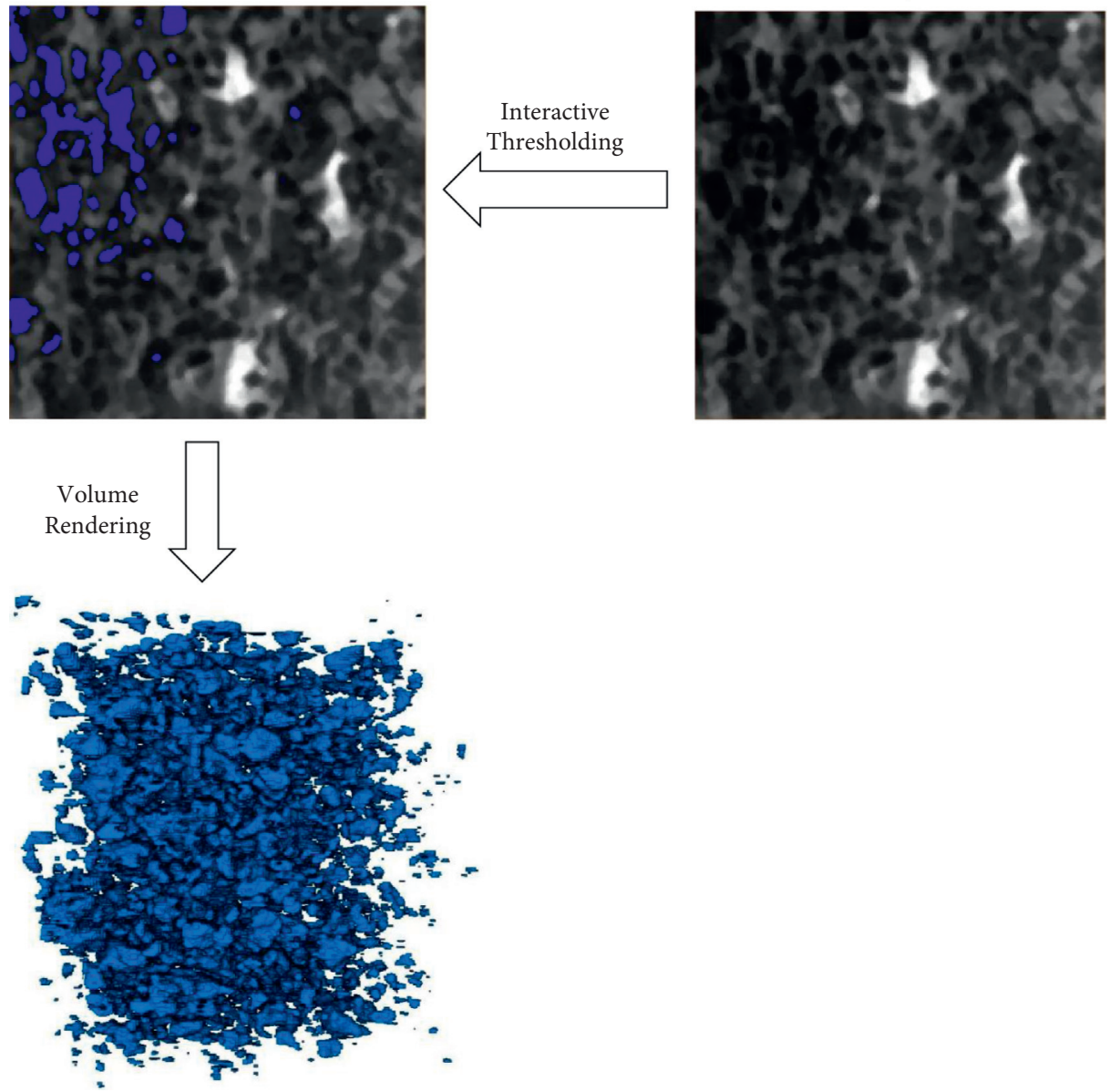

FIgURE 4: Three-dimensional pore extraction process.

biochemistry, and electrochemistry. According to the KC equation, the permeability $k$ and porosity $\varphi$ of the porous medium can be expressed as

$$
k=\frac{\phi^{3}}{c(1-\phi)^{2} S^{2}}=\frac{\phi^{3}}{36 c(1-\phi)^{2}} d^{2} .
$$

In the above formula, $c$ and $S$ are the Kozeny constant and the specific surface area of the solid phase, respectively. Considering the tortuosity effect (tortuosity $\tau$ ), the KC equation can be further expressed as $[35,36]$

$$
k=\frac{\varphi^{3}}{36 c(1-\varphi)^{2} \tau^{2}} d^{2} .
$$




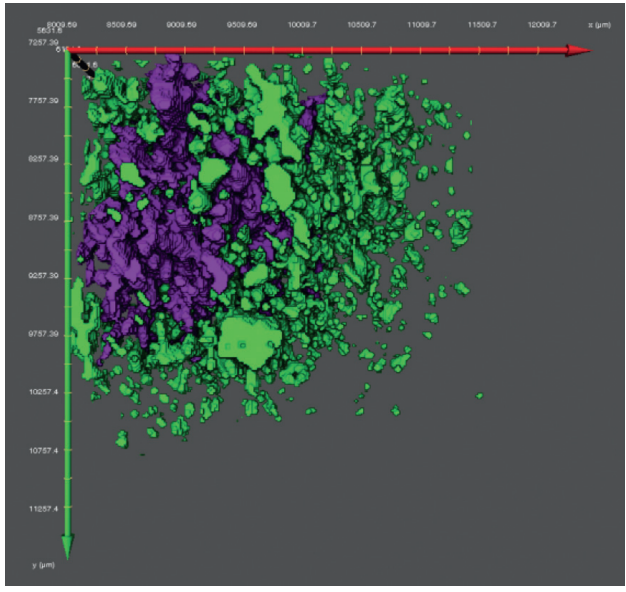

(a)

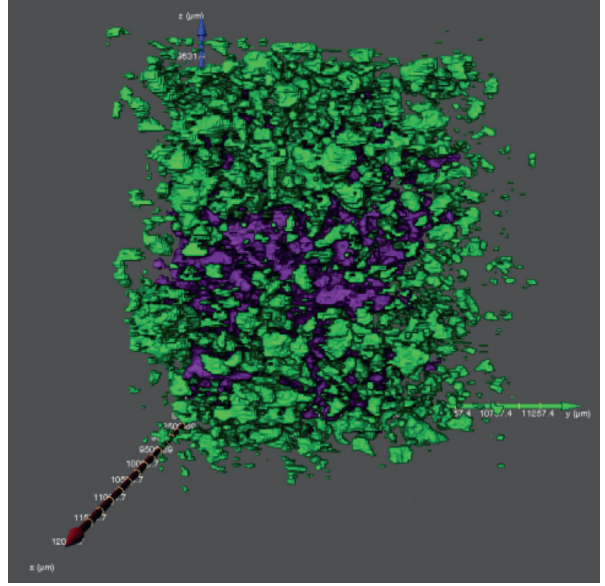

(b)

Figure 5: The perspective of total pores in the study area. (a) Total pores' perspective 1. (b) Total pores' perspective 2.

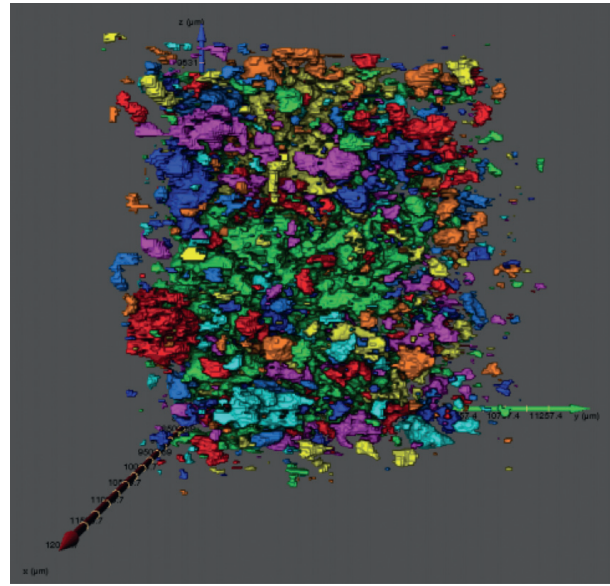

(a)

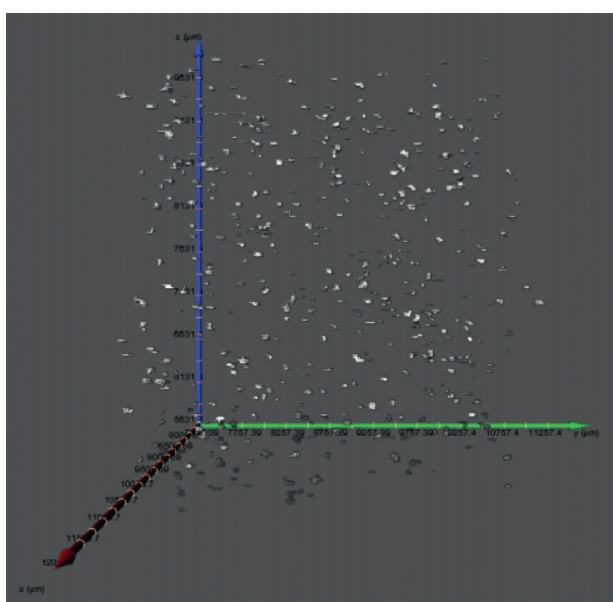

(c)

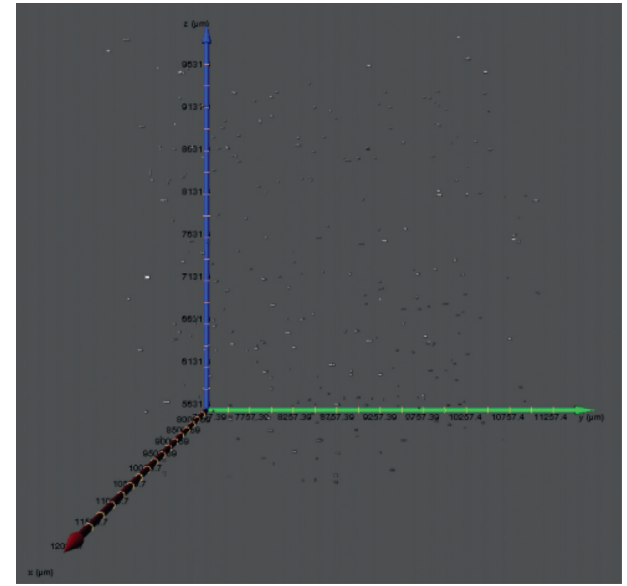

(b)

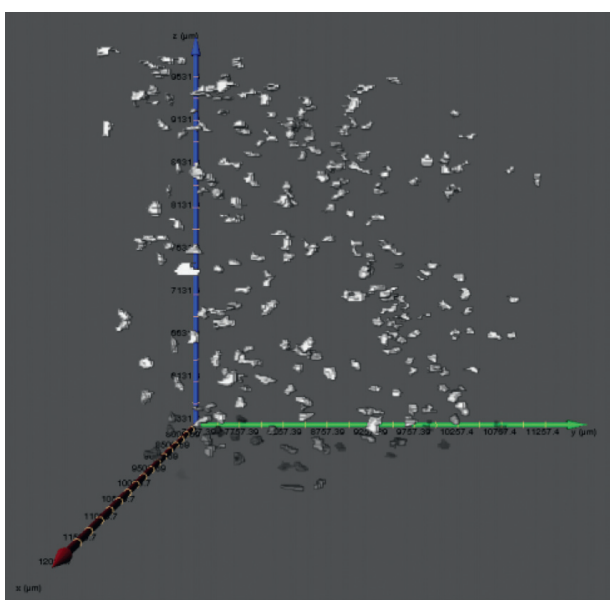

(d)

FIgURE 6: Continued. 


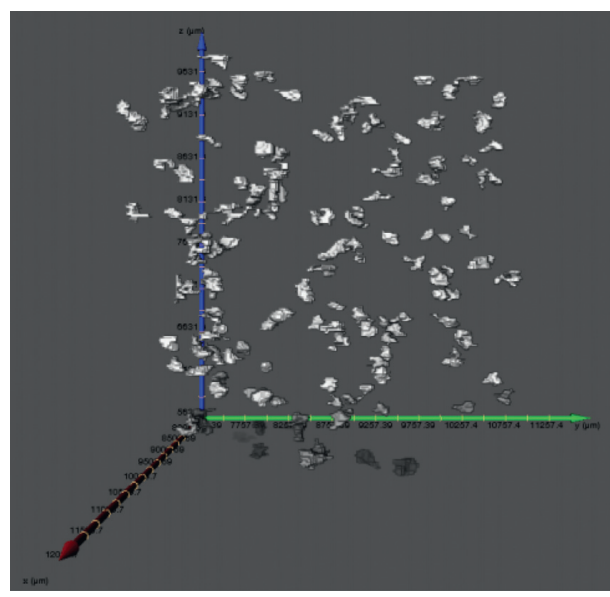

(e)



(g)

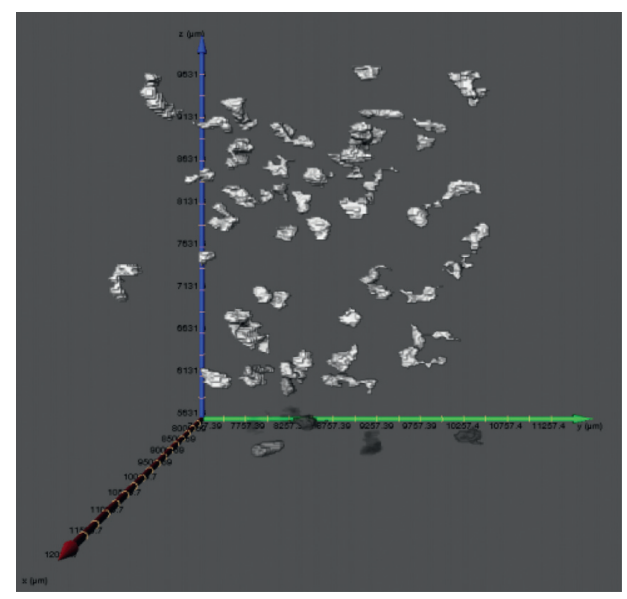

(f)

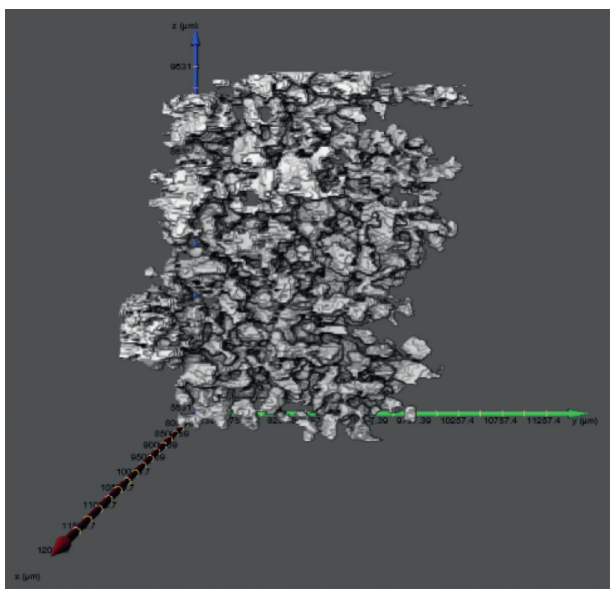

(h)

Figure 6: Aperture distribution diagram of total pores. (a) Total pores divided by equivalent diameter. (b) $0-40 \mu \mathrm{m}$ aperture. (c) $40-80 \mu \mathrm{m}$ aperture. (d) $80-120 \mu \mathrm{m}$ aperture. (e) $120-160 \mu \mathrm{m}$ aperture. (f) $160-200 \mu \mathrm{m}$ aperture. (g) 200-400 $\mu \mathrm{m}$ aperture. (h) Aperture greater than $400 \mu \mathrm{m}$.

TABLE 2: Pore type and porosity.

\begin{tabular}{lccccc}
\hline $\begin{array}{l}\text { Rock } \\
\text { sample }\end{array}$ & $\begin{array}{c}\text { Total porosity } \\
(\%)\end{array}$ & $\begin{array}{c}\text { Isolated porosity } \\
(\%)\end{array}$ & $\begin{array}{c}\text { Connected porosity } \\
(\%)\end{array}$ & $\begin{array}{c}\text { The overall proportion of isolated } \\
\text { pores (\%) }\end{array}$ & $\begin{array}{c}\text { The overall proportion of } \\
\text { connected pores }(\%)\end{array}$ \\
\hline GQ1 & 7.85 & 3.34 & 4.51 & 42.55 & 57.45 \\
GQ2 & 4.81 & 0.93 & 3.88 & 19.33 & 80.67 \\
GQ3 & 6.55 & 1.97 & 4.58 & 30.08 & 69.92 \\
TL1 & 6.54 & 0.94 & 5.6 & 14.37 & 85.63 \\
TL2 & 2.35 & 1.21 & 1.14 & 51.49 & 48.51 \\
LA1 & 2.51 & 2.13 & 0.38 & 84.86 & 15.14 \\
LA2 & 1.62 & 0.88 & 0.74 & 54.32 & 45.68 \\
\hline
\end{tabular}

Kaviany [36] believed that if the value of $t$ was approximately $21 / 2$, then for spherical particles, $c$ was 2.5 . Although the KC equation was widely used, people have noticed its limitations. The value of $c$ varied widely in different scenarios. Therefore, we $\operatorname{did}$ not consider $c$ as a key factor when establishing the permeability model.
4.2. Modification of the Kozeny-Carman (KC) Equation. Introducing a shape factor into the Kozeny-Carman equation and based on the traditional KC permeability prediction model, a permeability prediction model with shape factor as an influencing factor is established: 


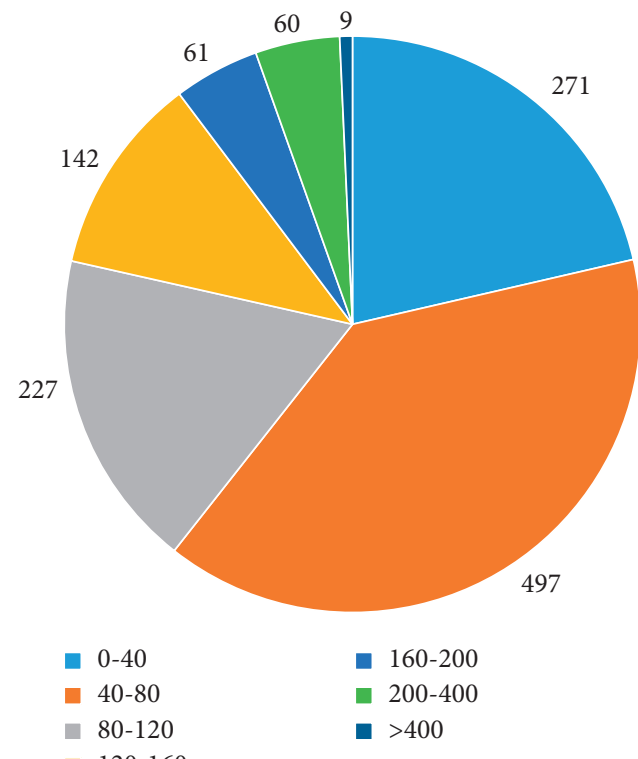

FIGURE 7: Distribution diagram of pores' size.
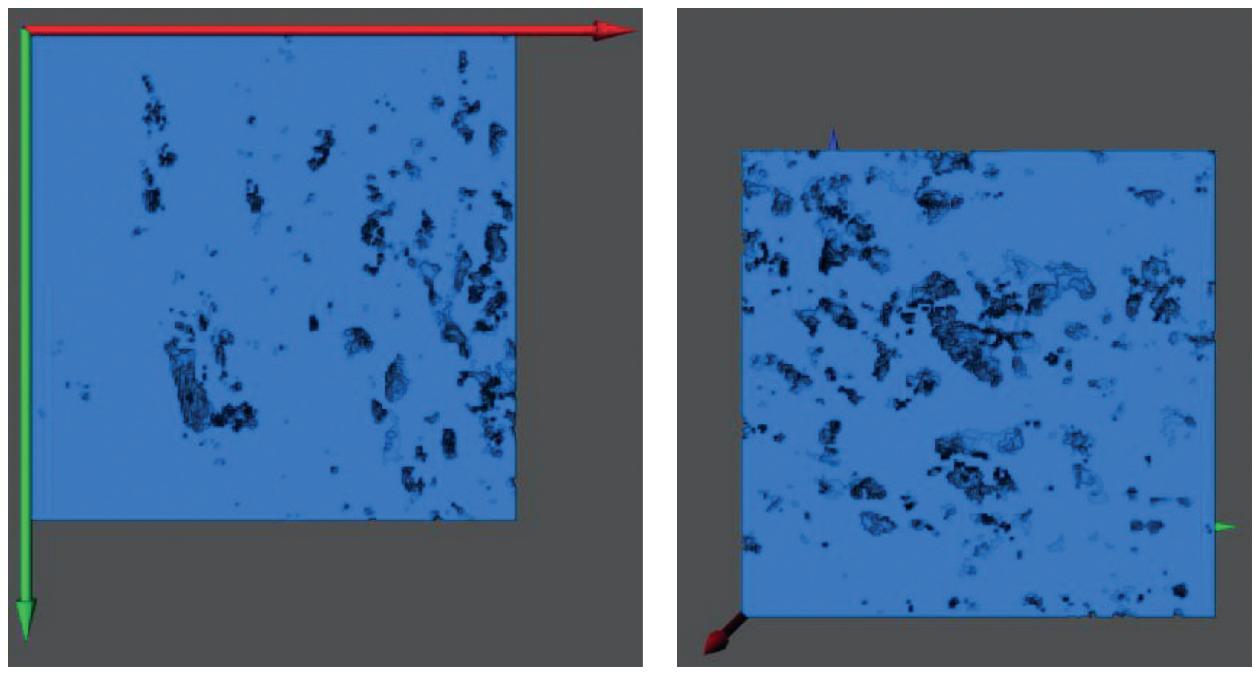

(a)

(b)

FIgURE 8: Continued. 


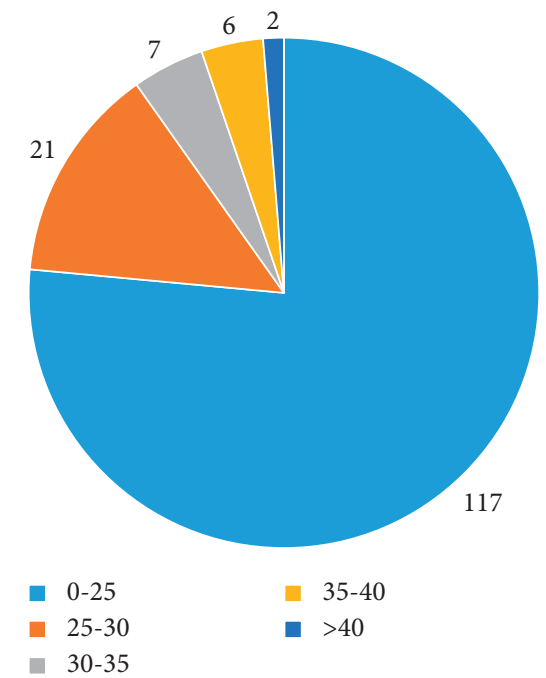

(c)

Figure 8: The particles in the study area. (a) Particles' perspective 1. (b) Particles' perspective 2. (c) Diameter of particles.

TABle 3: The diameter of pores and particles.

\begin{tabular}{lcc}
\hline Name of the rock sample & Average diameter of the connected pore $(\mu \mathrm{m})$ & Average diameter of the particle $(\mu \mathrm{m})$ \\
\hline GQ1 & 68.10 & 35.00 \\
GQ2 & 45.00 & 43.00 \\
GQ3 & 52.53 & 26.00 \\
TL1 & 63.30 & 49.77 \\
TL2 & 29.00 & 40.00 \\
LA1 & 34.30 & 28.50 \\
LA2 & 39.21 & 53.48 \\
\hline
\end{tabular}

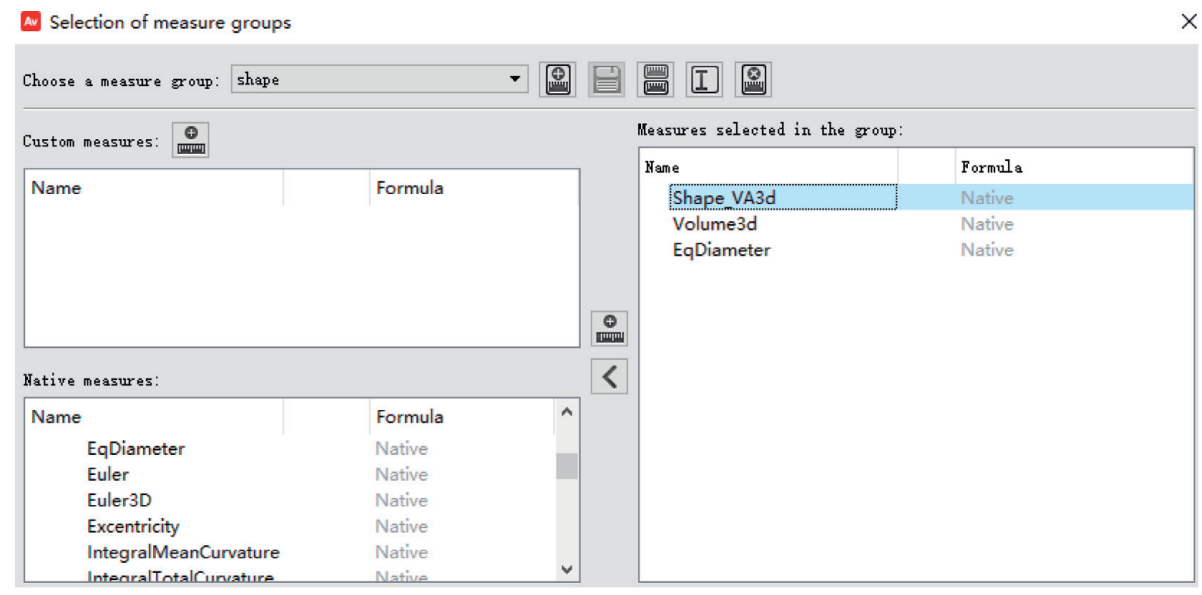

FIGURE 9: Shape factor module.

$$
k=\frac{\varphi^{3}}{36 \tau^{2}(1-\varphi)^{2} \sigma^{n}} d^{2},
$$

where $s$ is the pore shape factor and $n$ is the constant.

After analyzing and discussing the cores with different shape factors, it can be found that the exponent of the shape factor was \pm 15.8 . When the exponent of the shape factor was 15.8 for samples GQ2, GQ3, and LA2 and the exponent was
-15.8 for samples GQ1, TL1, TL2, and LA1, the errors of calculated permeability and experiment permeability were small. The obtained permeability prediction model is as follows:

$$
k=\frac{\varphi^{3}}{36 \tau^{2}(1-\varphi)^{2} \sigma^{ \pm 15.8}} d^{2} .
$$






(a)

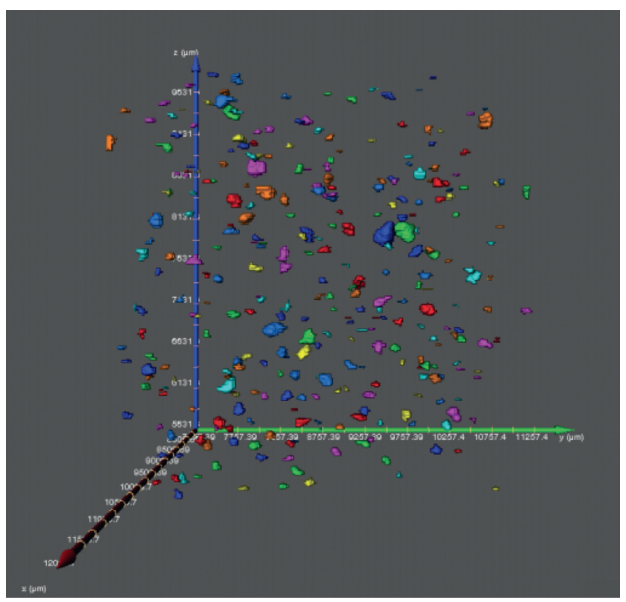

(c)

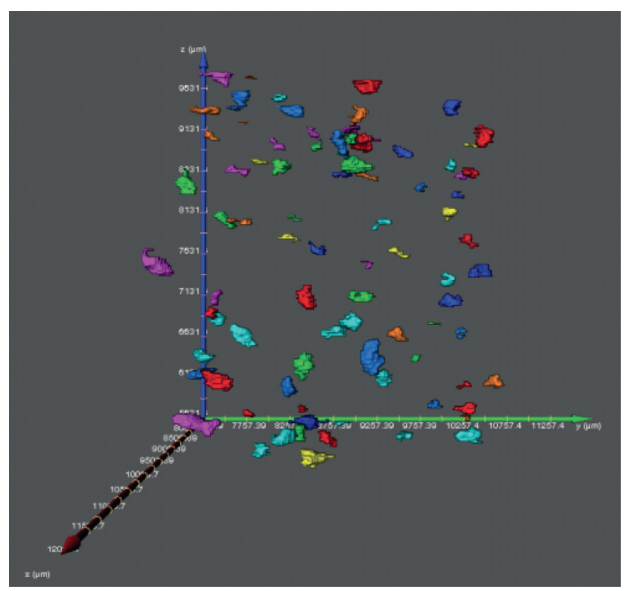

(e)

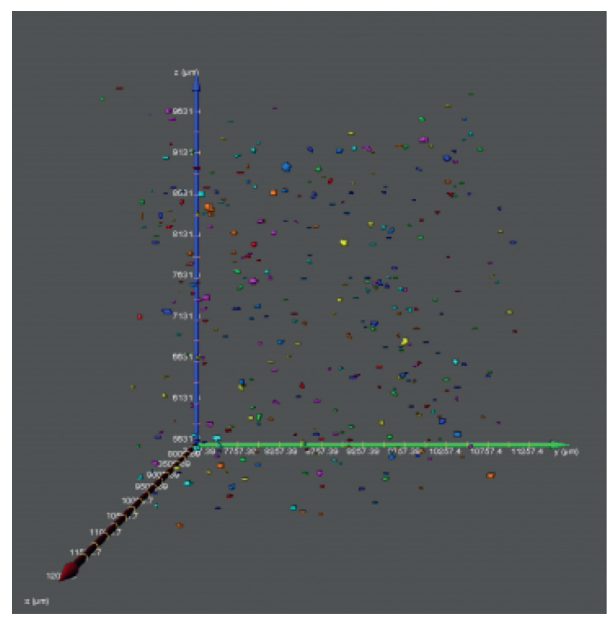

(b)

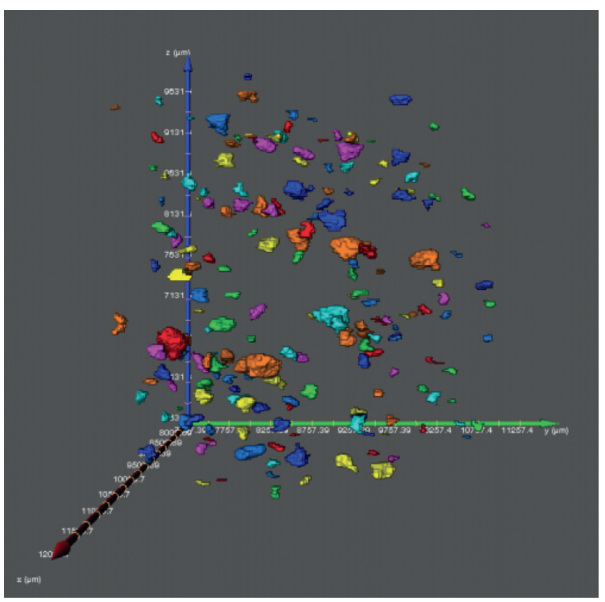

(d)

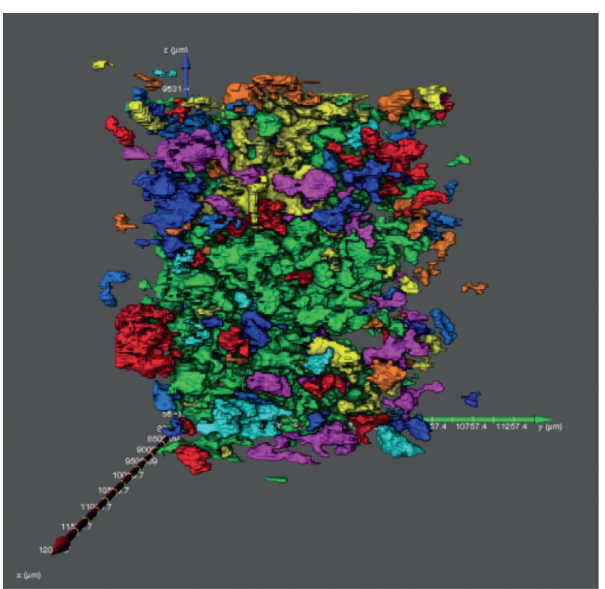

(f)

FIGURE 10: Distribution of GQ1's pore shape factor. (a) 0-0.5 shape factor section. (b) 0.5-1 shape factor section. (c) 1-1.5 shape factor section. (d) 1.5-2 shape factor section. (e) 2-2.5 shape factor section. (f) The section of shape factor greater than 2.5 .

Using formula (16), the theoretical permeability and relative error are calculated when $n$ is \pm 15.8 (as shown in Table 6).

The calculated permeability errors of the samples are within $15.6 \%$, and these errors are acceptable in engineering applications. Table 6 shows that shape factors have an effect on the permeability; however, since only seven samples were used, the data were small. This exponent may be different from the test samples, so the exponent values of \pm 15.8 require further investigation. 


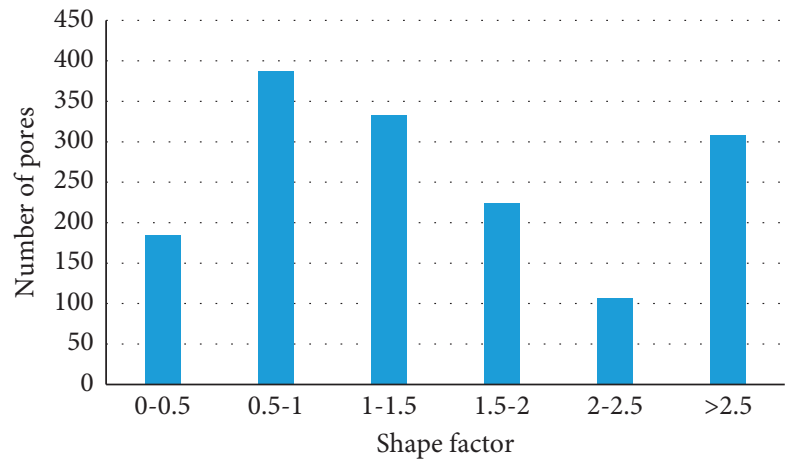

(a)

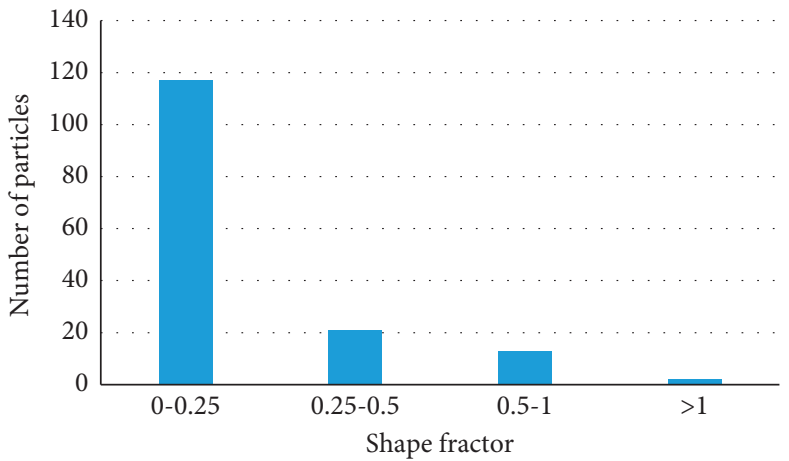

(b)

FIGURE 11: Statistics of GQ1's pore and particle shape factor. (a) Shape factor of pores. (b) Shape factor of particles.

TABLE 4: The shape factor of pores and particles.

\begin{tabular}{lcc}
\hline Name of the rock sample & Particles' shape factor & Pores' shape factor \\
\hline GQ1 & 1.24 & 2.03 \\
GQ2 & 0.80 & 1.69 \\
GQ3 & 0.82 & 2.01 \\
TL1 & 1.40 & 2.42 \\
TL2 & 0.83 & 0.59 \\
LA1 & 0.75 & 0.52 \\
LA2 & 1.20 & 0.59 \\
\hline
\end{tabular}

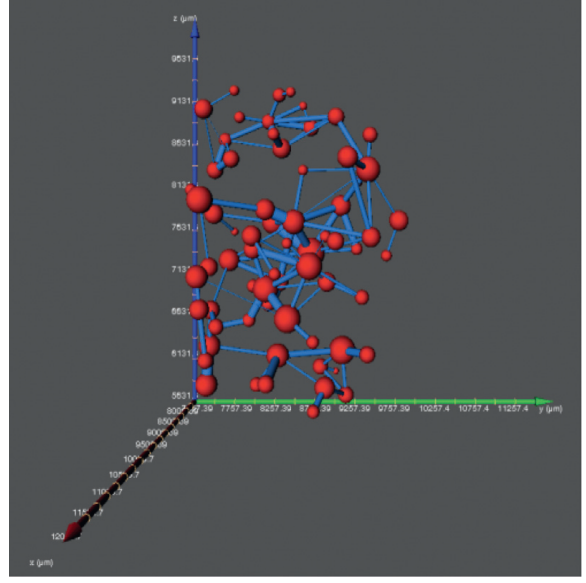

(a)

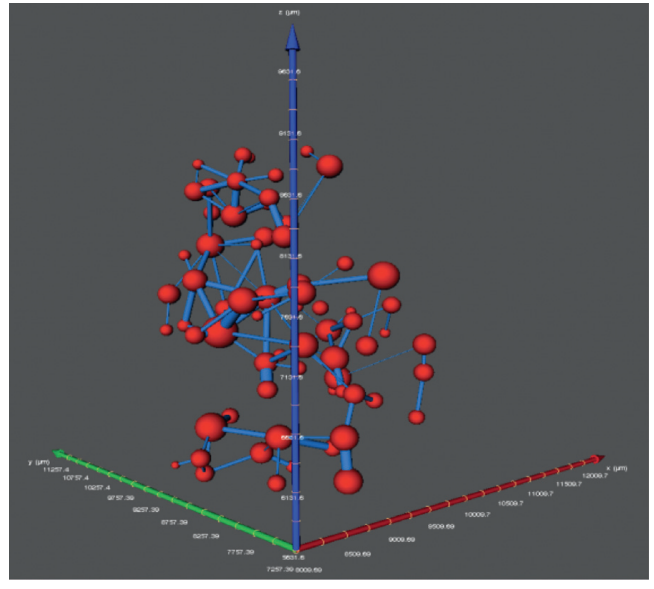

(b)

FIGURE 12: GQ1's ball-and-stick model. (a) Angle of view one of the ball-and-stick model. (b) Angle of view two of the ball-and-stick model.

TABLE 5: The tortuosity of pores.

\begin{tabular}{lr}
\hline Name of the rock sample & Pore tortuosity \\
\hline GQ1 & 2.93 \\
GQ2 & 3.17 \\
GQ3 & 3.22 \\
TL1 & 3.22 \\
TL2 & 5.50 \\
LA1 & 4.52 \\
LA2 & 6.02 \\
\hline
\end{tabular}


TABLE 6: Core permeability and relative error.

\begin{tabular}{lccc}
\hline Name of the rock sample & Actual permeability $(\mathrm{mD})$ & Theoretical permeability $(\mathrm{mD})$ & Relative error $(\%)$ \\
\hline GQ1 & 0.0144 & 0.0138 & 4.3 \\
GQ2 & 0.0102 & 0.0096 & 5.6 \\
GQ3 & 0.0084 & 0.0084 & 0.3 \\
TL1 & 0.0052 & 0.0067 & 7.9 \\
TL2 & 0.0870 & 0.0869 & 0.2 \\
LA1 & 0.0069 & 0.0058 & 15.6 \\
LA2 & 0.0160 & 0.0163 & 1.8 \\
\hline
\end{tabular}

\section{Conclusion}

This paper used X-ray CT scanning technology and Avizo software to study and calculate pore and particle structure parameters of low-permeability sandstones. Finally, the pore and particle structure parameters were used to predict permeability. In addition, the shape factor was introduced to modify the $\mathrm{KC}$ equation.

In this study, seven sandstone samples were collected from the Lu'an, Gequan, and Tunlan coal mines, China. The pore and particle geometric parameters of the samples were analyzed by CT experiments and Avizo software. The permeability and porosity were tested using the core company's high- and low-permeability meter CAT112 and a helium porosity meter from American Coretest company Phi220. The three-dimensional digital cores were analyzed by Avizo software, and a permeability prediction model was established. From these studies, the following conclusions may be drawn:

(1) The microstructures of the pore and particle can be well captured using CT experiments on sandstones and rock cores, and the structures of samples can be effectively obtained using Avizo software.

(2) Through experiments, it was found that the degree of correlation between porosity and permeability was not very high, and there were the cases of low porosity and high permeability.

(3) The parameters such as porosity, connected porosity, average equivalent diameter, tortuosity, and shape factor can be calculated by Avizo software from the extracted volume.

(4) It can be concluded that the shape factors have an effect on the permeability. By introducing the shape factor into the Kozeny-Carman equation, we modified the Kozeny-Carman equation.

\section{Data Availability}

The data used to support the findings of this study are included within the article.

\section{Conflicts of Interest}

The authors declare that they have no conflicts of interest.

\section{Acknowledgments}

This paper was partially supported by the Open Fund (PSLN1410) of State Key Laboratory of Oil and Gas
Reservoir Geology and Exploitation (Southwest Petroleum University) and supported by the National Science Foundation of China (51974126, 52174181, and 51204069) and Funding Projects for Basic Scientific Research Business Fees in Central Universities (3142015092 and 3142015093 ).

\section{References}

[1] Q. Xu, X. Liu, Z. Yang, and J. Wang, "The model and algorithm of a new numerical simulation software for low permeability reservoirs," Journal of Petroleum Science and Engineering, vol. 78, no. 2, pp. 239-242, 2011.

[2] M. A. Knackstedt, C. H. Arns, M. Saadatfar et al., "Elastic and transport properties of cellular solids derived from threedimensional tomographic images," Proceedings of The Royal Society A, vol. 462, no. 2073, pp. 2833-2862, 2006.

[3] W. Tian and N. Han, "Pore characteristics $(>0.1 \mathrm{~mm})$ of nonair entrained concrete destroyed by freeze-thaw cycles based on CT scanning and 3D printing," Cold Regions Science and Technology, vol. 151, pp. 314-322, 2018.

[4] J. Wang, J. Zhao, Y. Zhang, D. Wang, Y. Li, and Y. Song, "Analysis of the effect of particle size on permeability in hydrate-bearing porous media using pore network models combined with CT," Fuel, vol. 163, pp. 34-40, 2016.

[5] G. Wang, J. Shen, S. Liu, C. Jiang, and X. Qin, "Three-dimensional modeling and analysis of macro-pore structure of coal using combined X-ray CT imaging and fractal theory," International Journal of Rock Mechanics and Mining Sciences, vol. 123, Article ID 104082, 2019.

[6] Y. Cao, M. Tang, Q. Zhang, J. Tang, and S. Lu, "Dynamic capillary pressure analysis of tight sandstone based on digital rock model," Capillarity, vol. 3, no. 2, pp. 28-35, 2020.

[7] M. Qin, S. Xie, J. Zhang et al., "Petrophysical texture heterogeneity of vesicles in andesite reservoir on micro-scales," Journal of Earth Science, vol. 32, no. 4, pp. 799-808, 2021.

[8] Y. Yang, H. Yang, L. Tao et al., "Microscopic determination of remaining oil distribution in sandstones with different permeability scales using computed tomography scanning," Journal of Energy Resources Technology, vol. 141, Article ID 092903, 2019.

[9] H. Bian, Y. Xia, C. Lu, X. Qin, Q. Meng, and H. Lu, "Pore structure fractal characterization and permeability simulation of natural gas hydrate reservoir based on CT images," Geofluids, vol. 2020, Article ID 6934691, 9 pages, 2020.

[10] J. T. Fredrich, A. A. DiGiovanni, and D. R. Noble, "Predicting macroscopic transport properties using microscopic image data," Journal of Geophysical Research:Solid Earth, vol. 111, Article ID B03201, 2006.

[11] M. A. Mousavi and S. L. Bryant, "Connectivity of pore space as a control on two-phase flow properties of tight-gas sandstones," Transport in Porous Media, vol. 94, no. 2, pp. 537-554, 2012. 
[12] Y. Nakashima and S. Kamiya, "Mathematica programs for the analysis of three-dimensional pore connectivity and anisotropic tortuosity of porous rocks using X-ray computed tomography image data," Journal of Nuclear Science and Technology, vol. 44, no. 9, pp. 1233-1247, 2007.

[13] S. Tian, W. Ren, G. Li, R. Yang, and T. Wang, "A theoretical analysis of pore size distribution effects on shale apparent permeability," Geofluids, vol. 2017, Article ID 7492328, 26 pages, 2017.

[14] Y. Yang, Y. Li, J. Yao et al., "Formation damage evaluation of a sandstone reservoir via pore-scale X-ray computed tomography analysis," Journal of Petroleum Science and Engineering, vol. 183, Article ID 106356, 2019.

[15] K. Sueyoshi, T. Yokoyama, and I. Katayama, "Experimental measurement of the transport flow path aperture in thermally cracked granite and the relationship between pore structure and permeability," Geofluids, vol. 2020, Article ID 8818293, 10 pages, 2020.

[16] D. Hu and M. J. Blunt, "Pore-network extraction from microcomputerized-tomography images," Physical Review E, vol. 80, Article ID 036307, 2009.

[17] F. Feng, P. Wang, Z. Wei et al., "A new method for predicting the permeability of sandstone in deep reservoirs," Geofluids, vol. 2020, Article ID 8844464, 16 pages, 2020.

[18] W. Wei, J. Cai, J. Xiao, Q. Meng, B. Xiao, and Q. Han, "Kozeny-Carman constant of porous media: insights from fractal-capillary imbibition theory," Fuel, vol. 234, pp. 1373-1379, 2018.

[19] J. Kozeny, "Ueber kapillare Leitung des Wassers im Boden.Stizungsber," Akad Wiss Wiss Wien, vol. 136, pp. 1271-1306, 1927.

[20] P. C. Carman, "Fluid flow through granular beds," Transactions of the Institution of Chemical Engineers, vol. 15, pp. 150-167, 1937.

[21] P. C. Carman, Flow of Gases through Porous Media, Butterworths, London, UK, 1956.

[22] P. Mostaghimi, M. J. Blunt, and B. Bijeljic, "Computations of absolute permeability on micro-CT images," Mathematical Geosciences, vol. 45, no. 1, pp. 103-125, 2013.

[23] H. Pape, C. Clauser, and J. Iffland, "Variation of permeability with porosity in sandstone diagenesis interpreted with a fractal pore space model," Pure and Applied Geophysics, vol. 157, no. 4, pp. 603-619, 2000.

[24] F. Civan, "Scale effect on porosity and permeability: kinetics, model, and correlation," AIChE Journal, vol. 47, no. 2, pp. 271-287, 2001.

[25] A. Costa, "Permeability-porosity relationship: a reexamination of the Kozeny-Carman equation based on a fractal porespace geometry assumption," Geophysical Research Letters, vol. 33, no. 2, Article ID L02318, 2006.

[26] B. Yu, "Analysis of flow in fractal porous media," Applied Mechanics Reviews, vol. 61, no. 5, pp. 1239-1249, 2008.

[27] P. Xu and B. Yu, "Developing a new form of permeability and Kozeny-Carman constant for homogeneous porous media by means of fractal geometry," Advances in Water Resources, vol. 31, no. 1, pp. 74-81, 2007.

[28] C. Ö. Karacan, "Prediction of porosity and permeability of caved zone in longwall gobs," Transport in Porous Media, vol. 82, no. 2, pp. 413-439, 2010.

[29] Y. Xia, J. Cai, E. Perfect, W. Wei, Q. Zhang, and Q. Meng, "Fractal dimension, lacunarity and succolarity analyses on CT images of reservoir rocks for permeability prediction," Journal of Hydrology, vol. 579, Article ID 124198, 2019.
[30] P.-F. Shen, X.-S. Li, and G. Li, "Gas permeability and shape factor of natural sediments in the South China Sea," Journal of Natural Gas Science and Engineering, vol. 83, Article ID 103626, 2020.

[31] D. Metha and M. C. Hawley, "Wall effect in packed columns," Industrial and Engineering Chemistry Process Design and Development, vol. 8, pp. 280-286, 1969.

[32] J. Wu and B. Yu, "A fractal resistance model for flow through porous media," International Journal of Heat and Mass Transfer, vol. 50, no. 19-20, pp. 3925-3932, 2007.

[33] D. Nemec and J. Levec, "Flow through packed bed reactors: 1. Single-phase flow," Chemical Engineering Science, vol. 60, no. 24, pp. 6947-6957, 2005.

[34] M. Safari, R. Gholami, M. Jami, M. A. Ananthan, A. Rahimi, and W. S. Khur, "Developing a porosity-permeability relationship for ellipsoidal grains: a correction shape factor for Kozeny-Carman's equation," Journal of Petroleum Science and Engineering, vol. 205, Article ID 108896, 2021.

[35] J. Bear, Dynamics of Fluid in Porous Media, Elsevier, New York, NY, USA, 1972.

[36] M. Kaviany, Principles of Heat Transfer in Porous Media, Springer-Verlag, New York, NY, USA, 2nd edition, 1995. 